\section{CORONARY DISEASE}

Acute coronary syndromes:

presentation — clinical spectrum

and management

Keith A A Fox

Department of Cardiology, The Royal Infirmary, Edinburgh, UK infarction, through partial coronary obstruction and distal ischaemia with minor enzyme release (minimal myocardial injury), to nonocclusive thrombosis with normal cardiac enzymes (unstable angina) (table 1).

The distinction between acute myocardial infarction and minimal myocardial injury is of immediate practical importance as emergency reperfusion treatment is indicated for acute infarction but not for the remainder of the acute coronary syndromes. ${ }^{1}$ Acute infarction patients are identified by the combination of a typical clinical syndrome and electrocardiographic changes of ST elevation, new bundle branch block or posterior infarction. Such patients usually evolve $\mathrm{Q}$ waves and the release of cardiac enzymes with elevations to more than twice the upper limit of normal. In contrast, those with minimal myocardial injury do not have sustained ST segment elevation or the evolution of $\mathrm{Q}$ waves, and cardiac enzyme release is no more than twice the upper limit of normal. The terms "non-Q wave myocardial infarction" and "subendocardial myocardial infarction" refer to retrospective or pathological features and they are of little value as a guide to management in the acute clinical setting. It is impossible to characterise infarctions accurately into $Q$ wave and non- $Q$ wave at the time of presentation. In contrast, those with minimal myocardial injury/unstable angina can be defined at presentation and include individuals with a spectrum of electrocardiographic changes (table 1), but no features to indicate the need for immediate reperfusion. The management of such patients consists of anti-ischaemic treatment ( $\beta$ blockers, nitrates, calcium antagonists) in combination with antiplatelet treatment (aspirin, adenosine diphosphate antagonists, glycoprotein IIb/IIIa inhibitors) and antithrombin treatment (heparin and low molecular weight heparin) (fig 2).

The most frequently cited classification of patients with unstable angina is that proposed by Braunwald (table 2) and it describes the time course and mode of presentation. Relations have been demonstrated between classification categories and outcome, but this system excludes ECG information and it pre-dates modern enzymatic measures including troponins and the important prognostic information that both measures convey. Furthermore, the classification pre-dates current treatment strategies and hence we lack an evidence base upon which to differentiate management according to the Braunwald classification.

An alternative is to separate patients into high, intermediate, and low risk categories. These categories are important for the choice of pharmacological and interventional treatment as the relations between risks and outcome have been defined in trials. Risk status requires regular review, and documentation, as patients may change risk status as ECG, haemodynamic, and enzyme findings evolve. Specific prediction of cumulative risk can be derived from registry and trial data (that is, the six month risk of death/myocardial infarction or death/myocardial infarction/refractory an-
Correspondence to: Cardiology, The Royal Infirmary, Edinburgh email: k.a.a.fox@ed.ac.uk

Table 1 Acute coronary syndromes

\begin{tabular}{|c|c|c|}
\hline Clinical syndrome & ECG features & Enzyme features \\
\hline $\begin{array}{l}\text { - Acute myocardial } \\
\text { infarction (MI) }\end{array}$ & $\begin{array}{l}\text { ST elevation } \\
\text { New bundle branch block } \\
\text { ECG changes of posterior MI } \\
\text { Evolution of } Q \text { waves }\end{array}$ & $\begin{array}{l}>2 \times \text { upper limit of CK-MB, CK } \\
\text { Troponins } \mathrm{T}>0.2 \mathrm{ng} / \mathrm{dl} \\
\text { Troponin } \mathrm{I}^{\star}>1.0-1.5 \mathrm{ng} / \mathrm{dl}\end{array}$ \\
\hline $\begin{array}{l}\text { - Minimal myocardial } \\
\text { injury }\end{array}$ & $\begin{array}{l}\text { Aborted ST elevation MI } \\
\text { Transient ST elevation } \\
\text { ST depression } \\
\text { T inversion } \\
\text { Minor non-specific ECG changes }\end{array}$ & $\begin{array}{l}<2 \times \text { elevation CK-MB, CK } \\
\text { Troponins T } 0.01-0.2 \mathrm{ng} / \mathrm{dl} \\
\text { Troponin } \mathrm{I}^{\star} 0.1 \text { or } 0.4 \mathrm{ng} / \mathrm{dl} \text { to } \\
1.0-1.5 \mathrm{ng} / \mathrm{dl}\end{array}$ \\
\hline - Unstable angina & $\begin{array}{l}\text { Transient ST elevation } \\
\text { ST depression } \\
\text { T inversion } \\
\text { Minor non-specific ECG changes } \\
\text { Normal ECG }\end{array}$ & $\begin{array}{l}\text { CK-MB, CK below upper limit of } \\
\text { normal } \\
\text { Troponins } \mathrm{T}<0.01 \mathrm{ng} / \mathrm{dl} \\
\text { Troponin } \mathrm{I}^{\star}<0.1 \text { or } 0.4 \mathrm{ng} / \mathrm{dl}\end{array}$ \\
\hline
\end{tabular}

^Troponin I cut off values depend upon assay system 


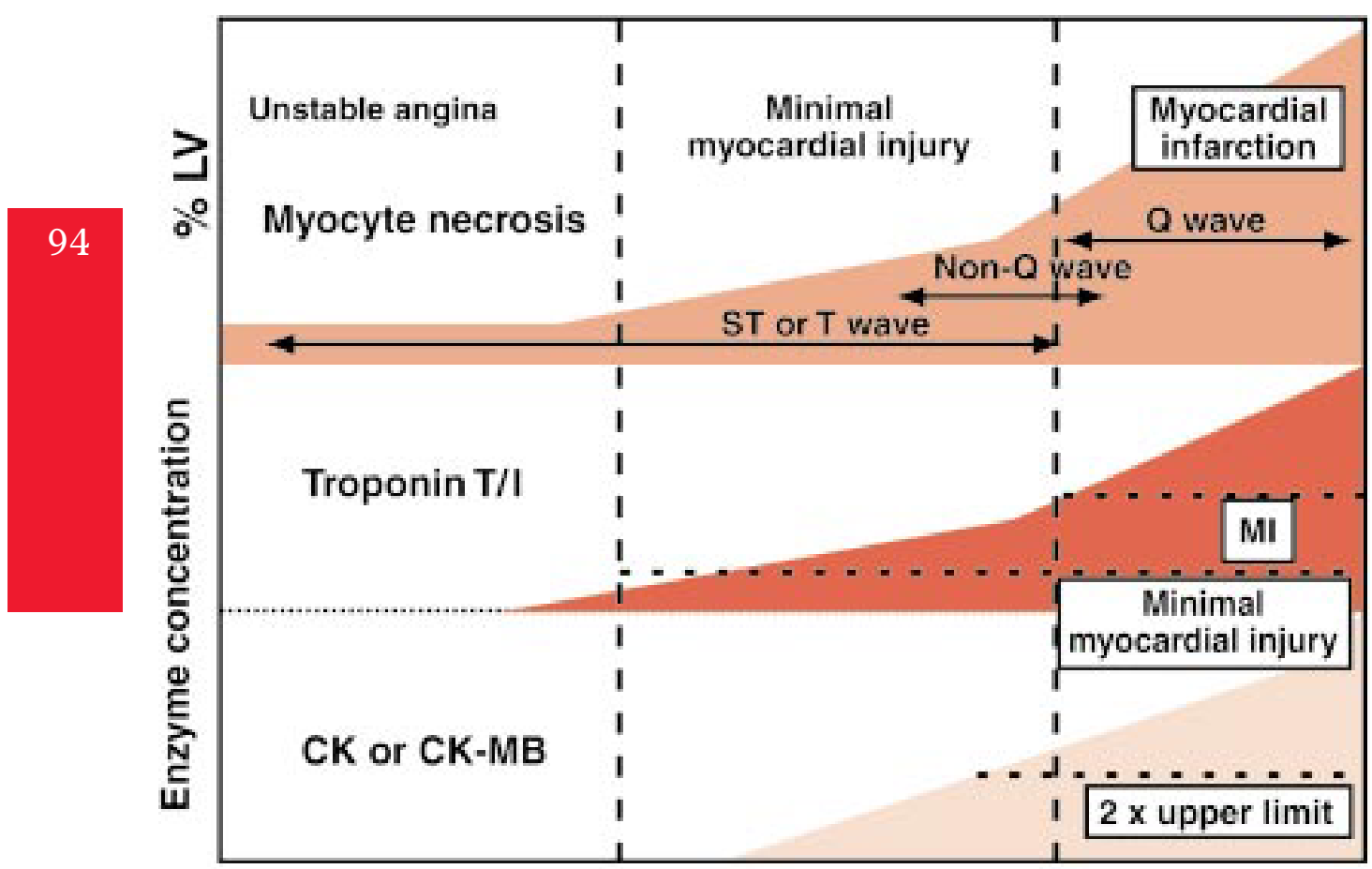

Figure 2: Spectrum of acute coronary syndromes. Schematic of the relation between the extent of myocardial necrosis, electrocardiographic markers, and cardiac enzymes (CK-MB and troponins). Troponins provide a more sensitive indicator of myocardial injury with a lower threshold indicating adverse prognostic outcome (troponin T $0.01 \mathrm{ng} / \mathrm{dl}$, troponin I $0.1-0.4 \mathrm{ng} / \mathrm{dl}$ depending on assay system), than for myocardial infarction (table 1).

gina), but for clinical purposes tertiles of risk are more straightforward (see below) as they do not require complex algorithms for their derivation.

Outcome: unstable angina and minimal myocardial injury

Previously, the hazards associated with unstable angina and minimal myocardial injury have been underestimated. Imprecise definitions of the syndrome and inconsistency in the need for corroborative evidence of acute coronary disease (enzyme markers and ECG change) have led to the inclusion of patients with chest pain and low prospective risk. For example, applying the Braunwald criteria, various forms of subacute rest pain, including that more than 48 hours previously, could classify the patient as class 2 unstable angina. This could include patients with entirely normal ECGs, normal cardiac enzymes, and low risk subsequent stress tests. Not surprisingly the outcome of such a heterogeneous population is more benign than for those with diagnostic features of the syndrome.

\section{Definition of the syndrome}

Based upon trial data and prospective registries the following features define patients with an acute coronary syndrome:

- Ischaemic chest pain (discomfort) at rest or on minimal exertion or emotion $(2 \times 5$ minute episodes or 1 episode $>10$ minutes). and

- Evidence of underlying coronary artery disease (at least one of the following):
- ECG: ST segment depression, $\mathrm{T}$ wave inversion or transient ST elevation

- Enzyme elevation: troponin I or T, creatine kinase $(\mathrm{CK})$ or $\mathrm{CK}-\mathrm{MB}$

- Evidence of coronary artery disease on angiography or perfusion scanning.

Patients with typical clinical features of unstable angina but a normal ECG and no prior documented coronary disease have a suspected acute coronary syndrome until enzymes and further ECGs confirm or refute the diagnosis.

Those with persistent ST elevation have suspected acute myocardial infarction and their management is considered elsewhere.

Overall outcome in unstable angina/non-ST elevation myocardial infarction

Based upon prospective international registry data among 8000 patients in six countries, the risk of death or myocardial infarction is approximately $10 \%$ at six months and almost a quarter of patients sustain these events or acute refractory angina within six months of initial presentation (OASIS registry) ${ }^{2}$ Overall, half of these events occur within the first seven days of presentation. Based on those included in clinical trials, and excluding those with normal ECGs, about $10 \%$ suffer death or myocardial infarction at 30 days (GUSTO II

Table 2 Braunwald classification of unstable angina

\begin{tabular}{lllll}
\hline Classification & $\begin{array}{l}\text { A. Secondary } \\
\text { unstable } \\
\text { angina }\end{array}$ & $\begin{array}{l}\text { B. Primary } \\
\text { unstable } \\
\text { angina }\end{array}$ & $\begin{array}{l}\text { C. Postinfarction } \\
\text { (<2 weeks) } \\
\text { unstable angina }\end{array}$ \\
\hline I & New onset, severe or accelerated angina & IA & IB & IC \\
II & Subacute rest angina ( $>48$ hours ago) & IIA & IIB & IIC \\
III & Acute rest angina (within 48 hours) & IIIA & IIIB & IIIC \\
\hline
\end{tabular}


data). ${ }^{3}$ These events occur despite aspirin treatment and antianginal medications. Recent data from the PRAISE UK registry indicate rates of death/myocardial infarction of $12.2 \%$ at six months. ${ }^{4}$

\section{Identification of high risk and low risk patients}

There are two main components to the risk carried by an individual patient: prior risk and acute ischaemic risk.

Prior risk is determined by systemic risk factors such as age, diabetes, hypertension, smoking, heart failure, and previous infarction. Such factors influence the extent of underlying coronary artery disease and left ventricular dysfunction, and their impact may be revealed by echocardiography, stress testing, perfusion scanning or coronary angiography.

Acute ischaemic risk is determined by the severity of impaired perfusion, the volume of myocardium affected, and the consequent changes in mechanical and electrical function. The distinction is important because a patient with a minor ischaemic event may nevertheless have extensive underlying coronary artery disease, and management strategies need to address both aspects of care. The converse may also occur.

The most powerful discriminators of acute ischaemic risk are:

- refractory angina with electrocardiographic evidence of ischaemia;

- ischaemia associated with haemodynamic instability or arrhythmia;

- recurrent ST segment change with positive troponin release;

- either positive troponin release or recurrent ST segment change.

A detailed discussion of risk prediction in acute coronary syndromes has been covered elsewhere. ${ }^{5}$ The key factors predicting adverse risk are summarised in the adjacent box. Readily available clinical characteristics can be used to separate patients into high, medium, and low risk based upon independent predictors of adverse outcome (prior risk characteristics and ECG changes). In consequence, the rates of death/myocardial infarction/stroke at six months are $7.4 \%, 10.1 \%$, and $17.9 \%$, respectively. ${ }^{2}$ Risk prediction is further improved by inducing troponin/CK-MB data and ST analysis.

Management of unstable angina and minimal myocardial injury

Presentation and general measures

Patients with an acute coronary syndrome may present de novo with new onset angina CCS (Canadian Cardiovascular Society) class III or IV, or following abrupt deterioration of previously stable angina with more severe and prolonged symptoms and diminished responsiveness to glyceryl trinitrate. The symptoms may be present at rest or may be precipitated by minor exertion or emotion. Where such symptoms develop within the first two weeks following acute myocardial inf-
Predicting adverse risk in unstable angina and minimal myocardial injury (summary)

- Prior risk older age ( $>65$ years)

- prior myocardial infarction or heart failure

- comorbidity: diabetes, hypertension - impaired renal function

Acute ischaemic risk

- refractory or recurrent ischaemic pain

- ECG: ST segment depression or transient ST elevation during pain ECG: T wave inversion (lower risk than ST segment depression or transient ST elevation)

- impaired left ventricular function with ischaemia

- release of cardiac enzymes: CK, $\mathrm{CK}-\mathrm{MB}$, troponin $\mathrm{T}$ or troponin I

- raised $\mathrm{C}$ reactive protein (high sensitivity assay)

arction, there is an increased risk of acute occlusion.

Patients with acute coronary syndromes may present directly to emergency departments (especially with acute infarction or severe ischaemia) but they may also present to chest pain clinics, care of the elderly units or to primary care physicians. On presentation a 12 lead ECG should be performed whenever possible during an episode of pain. This provides valuable diagnostic information.

Patients with diagnostic features of acute infarction or those of acute ischaemia with characteristic pain require emergency hospitalisation and management in a cardiac care unit or high dependency unit with continuous ECG monitoring. Repeat ECGs are required in those with suspected evolving infarction but in whom the initial features are nondiagnostic. Those with a suspected acute coronary syndrome should also be admitted directly to hospital (emergency admissions unit or chest pain assessment unit) to differentiate low risk patients for early discharge and intermediate/high risk patients for appropriate treatment. In current practice recent registry studies in the UK (PRAIS) ${ }^{4}$ and elsewhere $(\text { ENACT })^{6}$ suggest that low and high risk patients have similar lengths of stay; across Europe these averaged eight days of hospitalisation with approximately three days of care in a high dependency area. ${ }^{6}$ Patients in whom cardiac enzymes remain non-elevated at baseline and eight hours after presentation are at very low risk of subsequent cardiac events $(99 \%$ remain free of cardiac events, 95\% confidence interval $96 \%$ to $100 \%)$. Additional tests did not improve predictive accuracy. ${ }^{7}$ 
Presentation and diagnosis of acute coronary syndromes: summary

Ischaemic chest pain $>2 \times 5$ minutes or $>10$ minutes at rest or minimal exertion or persistent symptoms of myocardial infarction ( \pm autonomic features).

Evolving acute myocardial infarction - ECG: ST $\uparrow$, bundle branch block, posterior myocardial infarction Manage for acute myocardial infarction

- Abnormal ECG

- transient ST $\uparrow, S T \downarrow, T \downarrow$

Diagnosis: unstable angina/minimal myocardial injury

- elevated troponin T/I or CK, CK-MB Diagnosis: minimal myocardial injury

- enzymes not elevated

Troponin $\mathrm{T}<0.2$, troponin $\mathrm{I}<$ upper limit for laboratory

CK, CK-MB $<2 \times$ upper limit for lab

Diagnosis: unstable angina

ECG: persistence of previously abnormal ECG (conduction defect, Q waves, $\mathrm{T} \downarrow$ )

Diagnosis: suspected acute coronary syndrome

a) Repeat ECG, especially during pain b) Troponin $\mathrm{T} / \mathrm{I}$, or $\mathrm{CK}, \mathrm{CK}-\mathrm{MB}$ If (a) or (b) diagnostic: acute coronary syndrome confirmed.

If neither (a) nor (b) diagnostic: unstable angina or non-acute coronary syndrome diagnosis. Requires stress test \pm angiography to confirm or refute diagnosis.

- Normal ECG

ECG normal at baseline and 8-12 hours after pain. Troponins normal at 8-12 hours after pain.

Diagnosis: low risk patient or non-cardiac diagnosis

\section{Antiplatelet treatment}

\section{Aspirin}

The critical role of platelets and of thrombus formation in the pathophysiology of this condition is discussed elsewhere. ${ }^{8}$ Although aspirin is an irreversible inhibitor of platelet cyclooxygenase, and can inhibit the formation of thromboxane $\mathrm{A}_{2}$ and inhibit platelet aggregation, its effects can be overcome in the presence of potent thrombogenic stimuli. Nevertheless, the benefits of aspirin are substantial and clearly defined; the antiplatelet trialist collaboration demonstrated a $36 \%$ reduction in death or myocardial infarction with antiplatelet treatment (predominantly aspirin) versus placebo in unstable angina trials. ${ }^{9}$ Four key studies have demonstrated that aspirin almost halves the risk of cardiac death or non-fatal MI in patients with unstable angina. Thus, aspirin treatment is indicated in all patients with acute coronary syndromes unless there is good evidence of aspirin allergy. A starting dose of $300 \mathrm{mg}$ (chewed) and a maintenance dose of $75 \mathrm{mg}$ daily is recommended.

\section{Adenosine diphosphate antagonists}

The platelet adenosine diphosphate inhibitor clopidogrel has been employed as an adjunctive antiplatelet agent during coronary stenting. It appears to offer similar benefits to those of ticlopidine but with a more favourable safety profile (severe neutropenia as infrequent as that of aspirin: $0.04 \%$ clopidogrel $v 0.02 \%$ aspirin). Chronic treatment with clopidogrel offers approximately a $9 \%$ risk reduction compared with aspirin treatment and it is specifically indicated in those with aspirin intolerance. The use of combination aspirin and clopidogrel in acute coronary syndromes is currently under evaluation.

\section{Glycoprotein Ilb/Illa inhibitors}

Despite the undoubted benefits of aspirin, patients with acute coronary syndromes nevertheless suffer important risks of subsequent cardiac events. In the presence of a potent thrombogenic stimulus, like that which follows rupture of an atheromatous plaque, the effects of aspirin may be overcome and platelet aggregation ensues. Cross linking of platelets occurs via the glycoprotein IIb/IIIa receptor, with fibrinogen acting as the bridge. ${ }^{10}$

Large scale clinical trials have been conducted with three glycoprotein IIb/IIIa inhibitors: abciximab, tirofiban, and eptifibatide. More than 32000 patients have been randomised in clinical trials of glycoprotein IIb/IIIa inhibitors (16 trials) and a highly significant benefit is observed for the combined end point of death or myocardial infarction at 48 hours, 30 days, and six months. Overall, there are approximately 20 fewer events per thousand patients treated. ${ }^{11} \mathrm{~A}$ highly significant benefit is also observed on the combined end point of death/myocardial infarction or revascularisation. The net impact on mortality is modest and not observed at 30 days and beyond, except in a pooled analysis of abciximab trials. It is convenient to group glycoprotein IIb/IIIa inhibitors together, and undoubtedly there is a class effect, but there are biological and pharmacological differences between the agents and important differences in trial design when comparing studies. No direct head to head trials have been conducted.

Studies have been performed on the use of glycoprotein IIb/IIIa inhibitors in high risk groups, including those undergoing percutaneous intervention; these studies reveal more pronounced treatment effects than seen for the unstable angina population as a whole (27 fewer events per 1000 patients treated compared with 13 fewer events per 1000 treated for those studied where percutaneous intervention was not mandatory). In addition, post-hoc analyses have been conducted on the CAPTURE, PRISM PLUS, and PRISM studies and these indicate that almost all of the benefit is seen among patients with troponin release.

Thus, glycoprotein IIb/IIIa inhibitors are indicated in patients with elevated troponins 
and in whom percutaneous intervention is scheduled. Irrespective of revascularisation strategy, evidence supports the use of glycoprotein IIb/IIIa inhibitors in those with recurrent or refractory ischaemia (despite heparin and aspirin treatment) and in whom intervention is delayed or contraindicated.

\section{Antithrombin treatment}

Unfractionated heparin is widely used in the management of patients with unstable angina or minimal myocardial injury, although the evidence supporting its use in the absence of aspirin treatment is less robust than in the presence of aspirin. Maintaining accurate antithrombin control with unfractionated heparin is unpredictable because of plasma proteins binding, including that induced by acute phase proteins. There is reduced effectiveness in the presence of platelet rich and clot bound thrombin. Nevertheless, unfractionated heparin has formed the reference standard against which other antithrombins have been compared.

\section{Low molecular weight heparins}

The FRISC trial demonstrated that low molecular weight heparin is superior to placebo in aspirin treated patients.

Trials have also been conducted of low molecular weight heparin versus unfractionated heparin and two of these trials (ESSENCE and TIMI 11b, both using enoxaparin) have indicated superiority, with an absolute reduction of 30 events per 1000 patients treated (death/myocardial infarction/refractory angina). These benefits are seen without excess major bleeding but with some increase in minor bleeding including bruising at puncture sites. Other trials of low molecular weight heparins have not shown benefit over unfractionated heparin, but the overall conclusions are as follows:

- low molecular weight heparin is superior to placebo in aspirin treated patients;

- low molecular weight heparin is at least as effective as unfractionated heparin;

- low molecular weight heparin can be used in place of unfractionated heparin and with practical advantages.

- The use of low molecular weight heparin with intervention and/or glycoprotein IIb/ IIIa inhibitors is still being defined.

\section{Anti-ischaemic treatment}

The aim of anti-ischaemic treatment is to reduce myocardial oxygen demand and to induce vasodilatation and hence reduce ischaemia. Both antithrombotic treatment and mechanical revascularisation may also reduce ischaemia, and these treatments are considered separately.

\section{Nitrates}

Nitrates act predominantly by venodilatation and in higher doses by arteriolar dilatation;

\section{Anti-ischaemic treatment: summary}

The following conclusions are based upon pharmacologic and clinical trial evidence of anti-ischaemic treatment:

Patients with suspected acute coronary syndromes (without persistent ST elevation) should be initiated on a $\beta$ blocker (unless contraindicated) and a nitrate

In those with contraindications to $\beta$ blockers, a heart rate slowing calcium antagonist should be employed

The combination of calcium antagonist and $\beta$ blocker is superior to either agent alone

- In patients with recurrent ischaemia (with ECG abnormalities) despite anti-ischaemic treatment, urgent revascularisation should be considered rather than the addition of a third or fourth anti-ischaemic agent

hence they reduce preload and afterload, thereby decreasing oxygen demand. Large outcome trials have been conducted using nitrates in acute myocardial infarction but not in the remainder of acute coronary syndromes. Their major limitation is the induction of tolerance, and increased doses of nitrates may be required with dose titration on the basis of the heart rate and blood pressure response, and relief of symptoms. Following the acute phase, patients may be switched to oral nitrates, but if tolerance has been induced such treatment may have reduced effectiveness.

\section{Calcium entry blockers}

Calcium antagonists act by inhibiting the slow inward current induced by the entry of extracellular calcium through the cell membrane. They lower myocardial oxygen demand and reduce arterial pressure and contractility. Some agents induce a reflex tachycardia and these are best administered in combination with a $\beta$ adrenoceptor antagonist. In contrast, diltiazem and verapamil are suitable for patients who cannot tolerate a $\beta$ blocker because they slow conduction through the atrioventricular node and tend to cause bradycardia. Calcium entry blockers have been shown to reduce the frequency of angina. A meta-analysis of calcium entry blockers in acute coronary syndromes indicates a nonsignificant trend towards higher mortality versus control patients $(5.9 \%$ v $5.2 \%$ in 7551 patients). In individual trials, diltiazem has been compared with propanolol and both agents produced a similar reduction in anginal episodes. In summary, patients unable to tolerate $\beta$ blockers should have a heart rate slowing calcium antagonist. Short acting dihydropyridines should not be used in isolation in acute coronary syndromes. 


\section{$\beta$ Blockers}

$\beta$ Adrenoceptor antagonists reduce heart rate, blood pressure, and myocardial contractility. They are mainly used to reduce ischaemia, and large scale outcome trials have not been conducted in unstable angina. A meta-analysis of five trials involving 4700 patients with threatened myocardial infarction (treated with intravenous $\beta$ blockers followed by oral therapy) resulted in approximately $13 \%$ reduction in the risk of myocardial infarction. In summary, $\beta$ blockers are the antianginal agents of choice in those without contraindications.

\section{Potassium channel activators}

Potassium channel activators (for example, nicorandil) have both arterial and venous dilating properties and do not exhibit the same tolerance as seen with nitrates. They have been shown to be better than placebo in relieving symptoms of angina, but little evidence exists in comparison with other antianginal agents. Nicorandil possesses both potassium channel and nitrate like properties and may be considered as an alternative to nitrate administration.

\section{Revascularisation}

Revascularisation may be required in the acute phase on account of refractory or recurrent symptoms; it may also be required following stabilisation in high risk patients (those with troponin release and/or ST segment depression). In addition, non-high risk patients should undergo stress testing during the recovery phase in order to detect those with severe underlying coronary artery disease. Such patients may also require revascularisation for prognostic indications (those with left main or three vessel disease, or severe two vessel disease and impaired left ventricular function). In addition, revascularisation may be required for the relief of symptoms in those in whom medical treatment proves inadequate.

Registry studies have demonstrated that although consistency exists for some aspects of management of patients with acute coronary symptoms, wide discrepancies occur from hospital to hospital and regionally with respect to revascularisation. ${ }^{26}$ Prospective registry studies have demonstrated that countries or regions with high revascularisation rates do not necessarily have improved outcomes compared with countries with lower revascularisation rates. ${ }^{2}$ Higher rates are associated with more periprocedural complications including stroke and bleeding. Counter-intuitively, most procedures are performed in lower risk rather than higher risk patients.

Limited randomised trial data exist. Trials in the 1970 s and 1980 s of coronary artery bypass surgery, in patients admitted with unstable angina, produced inconclusive results, and one of the two trials was non-randomised by design. The TIMI IIIb trial conducted in the early 1990s randomised 1473 patients to an early invasive or an early conservative strategy. However, it was rather underpowered and suf- fered from high crossover rates from the conservative to the invasive strategy $(61 \%$ revascularisation in the invasive arm versus $49 \%$ in the conservative arm). Death or myocardial infarction occurred in $7.2 \%$ of patients in the invasive arm versus $7.8 \%$ in the conservative arm (six weeks) and the corresponding rates at one year were $10.8 \%$ versus $12.2 \%$, with both comparisons being nonsignificant. The invasive strategy was associated with a lower rate of rehospitalisation.

In the VANQWISH trial there were 916 patients with evolving non-ST segment elevation myocardial infarction randomised to an aggressive or a more conservative strategy. These patients had a high prevalence of comorbidity and the death/reinfarction rate was $24 \%$ in the revascularisation group at one year versus $19 \%$ in the medical group. The excess mortality was primarily seen in those randomised to surgical revascularisation, but a substantial number of the deaths occurred in patients in whom the procedure was not performed. Nevertheless, the conclusions of the study suggested a net hazard with more aggressive surgical revascularisation.

In the FRISC II trial an effective separation of treatment strategies was achieved. Patients were stabilised on low molecular weight heparin for six days, and revascularisation performed in $71 \%$ of those in the invasive arm and only $9 \%$ in the non-invasive arm, within 10 days. At six months, death or myocardial infarction occurred in $9.4 \%$ of the invasive group compared to $12.1 \%$ of the non-invasive group (a risk ratio of $0.78, \mathrm{p}=0.031$ ), and the results remained significant at one year. Greatest benefits were demonstrated in those with the most pronounced ST segment change. However, the risk ratios were no greater for those with troponin release than those without.

In conclusion, taking all the trial data, the findings are not consistent. However, caution must be exercised in comparing older trials with more modern treatment strategies. FRISC II does provide evidence of benefit with revascularisation following an early period of stabilisation, but the findings need confirmation in other large trials (TACTICS and RITA-3). FRISC II has not tested aggressive early revascularisation (that is, within 72 hours) and the results should not be interpreted as such. Furthermore, the use of glycoprotein IIb/ IIIa inhibitors was low and adjunctive treatment may further reduce complications.

\section{Integrated approach to the}

management of unstable angina, minimal myocardial injury

There are three components:

- Identification of patients with suspected acute coronary syndromes.

- Establishing the diagnosis and risk category.

- Management.

Patients with a suspected acute coronary syndrome may present to their primary care physician, a hospital based emergency receiving unit (including care of the elderly) or an 


\section{Management of unstable angina/minimal myocardial injury}

All patients: aspirin, antianginal treatment, heparin/low molecular weight heparin, oxygen if required, manage arrhythmic and mechanical complications.

\section{Highest risk \\ Refractory angina with ischaemic ECG changes \\ - Ischaemia with haemodynamic instability or arrhythmia \\ - Recurrent ECG change and troponin elevation}

Management: Optimise anti-ischaemic treatment. Heparin/IV glycoprotein IIb/IIIa inhibitor. Heparin. Emergency angiography/revascularisation unless contraindicated or patient unfit to transfer

\section{High risk}

- Troponin elevation (or CK, CK-MB) but no ST elevation nor new $\mathrm{Q}$ waves. \pm ECG change (ST $\downarrow$ or $\mathrm{T} \downarrow$ )

Management: Optimise anti-ischaemic treatment. Heparin/low molecular weight heparin. Consider glycoprotein IIb/IIIa inhibitor

Clinically stable: pre-discharge angiography if candidate for revascularisation

If recurrent symptoms or haemodynamically unstable, see highest risk above

\section{Intermediate or indeterminate risk}

- Recurrent symptoms without ECG change Consider alternative diagnoses

Stress testing or angiography

- Persistence of previously abnormal ECG

Management: stress test - perfusion/echolexercise

tolerance test

- reversible perfusion defect/ischaemia: consider revascularisation

- no defect: alternative diagnosis

- fixed defect: prior myocardial infarction

\section{Low risk}

- Clinically stable, normal ECG, normal troponins ( $>12$ hours after pain)

Management: Discharge plus elective stress test (pre- or postdischarge)

acute cardiology unit. A clinical history of ischaemic chest pain is central to establishing the diagnosis. As a minimum, chest pain is present for at least two, five minute episodes or one, 10 minute episode at rest or on minimal exertion or emotion. In those with evolving infarction the pain may be persistent and accompanied by autonomic features.

\section{Trial acronyms}

CAPTURE: Chimeric 7e3 AntiPlatelet Therapy in Unstable angina Refractory to standard treatment

ENACT: European Network for Acute

Coronary Treatment

ESSENCE: Efficacy and Safety of

Subcutaneous Enoxaparin in unstable angina and Non-Q wave myocardial infarction

FRISC: FRagmin during InStability in Coronary artery disease

GUSTO: Global Use of Strategies To open Occluded coronary arteries

OASIS: Organisation to Assess Strategies

for Ischaemia Syndromes

PRAIS: Prospective Registry of Acute

Ischaemic Syndromes

PRISM: Platelet Receptor Inhibition for

Ischaemic Syndrome Management

RITA: Randomised Intervention Treatment of Angina Trial

TACTICS: Treat Angina with Aggrastat (tirofiban) and determine Cost of Therapy with Invasive or Conservative Strategy

TIMI: Thrombolysis In Myocardial

Infarction

VANQWISH: Veterans Affairs Non-Q Wave Infarction Strategies in Hospital

Following identification of those with suspected acute coronary syndrome, such patients are further categorised on the basis of their clinical syndrome plus the ECG changes, cardiac enzyme markers, and stress testing. This allows the identification of those with evidence of evolving acute infarction, those with suspected or confirmed unstable angina/minimal myocardial injury, and low risk patients, and those with noncardiac or non-acute coronary syndrome diagnoses.

\section{Conclusions}

Previously, the hazards of acute coronary syndromes (especially unstable angina or minimal myocardial injury) have been underestimated. This is mainly because of inconsistencies in diagnosis and the inclusion of patients with chest pain but without confirmatory evidence of an acute coronary syndrome.

Recent data from large scale clinical trials, and from registry studies, demonstrate that patients can be identified on the basis of the clinical syndrome plus electrocardiographic and enzyme criteria. These tools should be available in all hospitals.

Characterisation of patients with acute coronary syndromes firstly identifies those with suspected evolving acute infarction, for reperfusion treatment. Among the remainder, those with unstable angina or minimal myocardial injury are identified on the basis of ECG 
abnormalities or cardiac enzyme elevation, or both. This strategy allows the separation of high, intermediate, and low risk patients. Such stratification permits the targeting of more potent pharmacological treatment at those at highest risk, and the identification of patients with the most to gain from revascularisation strategies. Registry studies across Europe currently show that such stratification is not systematically performed, and that it does not currently guide management strategies. The above strategy has the further advantage that it allows the separation of low risk patients for early discharge. Thus diagnostic and risk stratification is based upon the underlying pathophysiology of the syndrome, it is validated in prospective clinical trials and registry studies, and it provides a rational basis for pharmacological and interventional treatment.

1. White HD. Unstable angina. In: Topol EJ, ed. Comprehensive cardiovascular medicine. Philadelphia: Lippincott-Raven, 1998.

- Comprehensive review of acute coronary syndromes.

2. Yusuf S, Flather M, Pogue, et al for the OASIS Registry Investigators. Variations between countries in invasive cardiac procedures and outcomes in patients with suspected unstable angina or myocardial infarction without initial ST elevation. Lancet 1998;352:507-14.

- OASIS registry study demonstrating risks of death, myocardial infarction, and recurrent angina among patients admitted with stable angina or non-ST elevation myocardial infarction.

3. The Global Use of Strategies to Open Occluded Coronary Arteries (GUSTO Ilb) Investigators. A

comparison of recombinant hirudin with heparin for the treatment of acute coronary syndromes. $N$ Engl J Med

1996;335:775-82.

- Large scale trial comparing hirudin and heparin in acute coronary syndromes and providing outcome data for this population.

4. Collinson J, Flather MD, Fox KAA, et al for the PRAIS-UK Investigators. Clinical outcomes, risk stratification and practice patterns of unstable angina and myocardial infarction without ST elevation: prospective registry of acute ischaemic syndromes in the UK (PRAIS-UK). Eur Heart $J$ In press.

- Prospective UK registry of unstable angina and non-ST elevation myocardial infarction. $12.2 \%$ rate of death/myocardial infarction at six months and $30 \%$ rate of death/myocardial infarction, refractory angina or readmission for unstable angina.

5. Timmis A. Acute coronary syndromes: risk stratification. Heart 2000;83:241-6.

- Education in Heart series article on risk stratification in acute coronary syndromes.

6. Fox KAA, Cokkinos DV, Deckers JW, et al. The ENACT study: a pan-European survey of acute coronary syndromes. Eur Heart $J$ In press.

- The first pan-European data on the management and in-hospital events for patients admitted with the full spectrum of acute coronary syndromes.

7. Hillis GS, Zhao $\mathbf{N}$, Taggart $\mathbf{P}$, et al. Utility of cardiac troponin I, creatine kinase- $\mathrm{MB}_{\text {mass }}$, myosin light chain 1 , and myoglobin in the early in-hospital triage of "high risk" patients with chest pain. Heart 1999;82:614-20.

- The role of specific cardiac enzymes in the triage of high risk patients with chest pain.

8. Davies MJ. The pathophysiology of acute coronary syndromes. Heart 2000;83:361-6.

- Education in Heart series on the pathophysiology of acute coronary syndromes.

9. Anti-platelet Trialists Collaboration. Collaborative overview of randomised trials of antiplatelet therapy - I: prevention of death, myocardial infarction, and stroke by prolonged antiplatelet therapy in various categories of patients. BMJ 1994;308:81-106.

- Key combined analysis of all of the trials of antiplatelet therapy up to 1994. Major impact of aspirin demonstrated.

10. Fox KAA. Comparing trials of glycoprotein Ilb/Illa receptor antagonists. Eur Heart J 1999;1(suppl R):R10-17.

- Review of the trials of glycoprotein Ilb/Illa antagonists for acute coronary syndromes.

11. Kong DF, Califf RM, Miller DP, et al. Clinical outcomes of therapeutic agents that block the platelet glycoprotein Ilb/llla integrin in ischemic heart disease. Circulation 1998;98:2829-35.

- Pooled analysis of the trials of glycoprotein IIb/IIla inhibitors for acute coronary syndromes with or without mandated intervention. Sustained impact on myocardial infarctions, especially in those undergoing percutaneous intervention. 